\author{
Wladyslaw Koc \\ prof.dr hab.inż. \\ Politechnika Gdańska \\ wladyslaw.koc@pg.gda.pl
}

\title{
The application of nonlinear curvature sections in the turnout diverging track
}

\begin{abstract}
The paper presents the analytical method of modelling the diverging track of railway turnout with nonlinear curvature sections. These sections were used for smoothing the graph of curvature in the extreme areas of turnout. The problem of the curvature distribution was identified with the use of differential equations. The resulting solutions are of universal nature for example the ability of assuming any values of curvature at the beginning and ending of the layout. The comparative analysis was performed for two cases with nonlinear curvature and corresponding cases where linear curvature was used. As a result, the analysis of selected cases showed a beneficial curvature distribution in a nonlinear variant, the extreme areas of circular arc are smoothed as well as the curvature at both ends of the layout is equal to zero.
\end{abstract}

Keywords: Railway turnouts; Curvature modelling; Analysis of horizontal ordinates

\section{Introduction}

In a typical geometrical configuration of the return path in railway displacement called ordinary, a single circular arc is used without transition curves. This solution is not used on railway routes and means the need to limit the speed of trains. This is due to the occurrence of places of rapid, abrupt change in the ordinates of the curvature diagram at the beginning and the end of the turnout. In recent years, in some countries, in particular on high-speed railways, the aim is to soften the curvature diagram in these regions. This is achieved by introducing the so-called "Clothoid sections" on both sides of a circular arc, on which the curvature varies in a linear manner, but often without reaching zero values in the end points $[2,8,9,10]$.

In the case of standard turnouts, the change of the operated switchgear to a turnout with a larger radius in the reverse path leads most often to the need to increase track gauge. In the case of switches with variable curvature - due to the individual nature of their design, it is possible to adjust the given turnout to local requirements. This is particularly important in parallel tracks connections, where the main issue is maintaining the proper track gauge.

The length of the return path is divided into three zones here (Figure 1):

an initial zone of length $l_{1}$, having a linear curvature,

a middle zone with length $l_{2}$, having a fixed curvature,

an end zone of length $l_{3}$, having a linear curvature.

Of course, different variants are possible here, related to the curvature values and the lengths of individual zones.

In the starting zone, forl $\in\left\langle 0, l_{1}\right\rangle$, the curvature grows linearly from $k_{1}=\frac{1}{R_{1}}$ (or $k_{1}=0$ ) to $k_{2}=\frac{1}{R_{2}}$, in the middle zone, for $l \in\left\langle l_{1}, l_{1}+l_{2}\right\rangle$, is constant and equals $k_{2}=\frac{1}{R_{2}}$, whereas in the end zone, for $l \in\left\langle l_{1}+l_{2}, l_{1}+l_{2}+l_{3}\right\rangle$, linearly decreases from $k_{2}=\frac{1}{R_{2}}$ to $k_{3}=\frac{1}{R_{3}}$ (or $\left.k_{3}=0\right)$. 


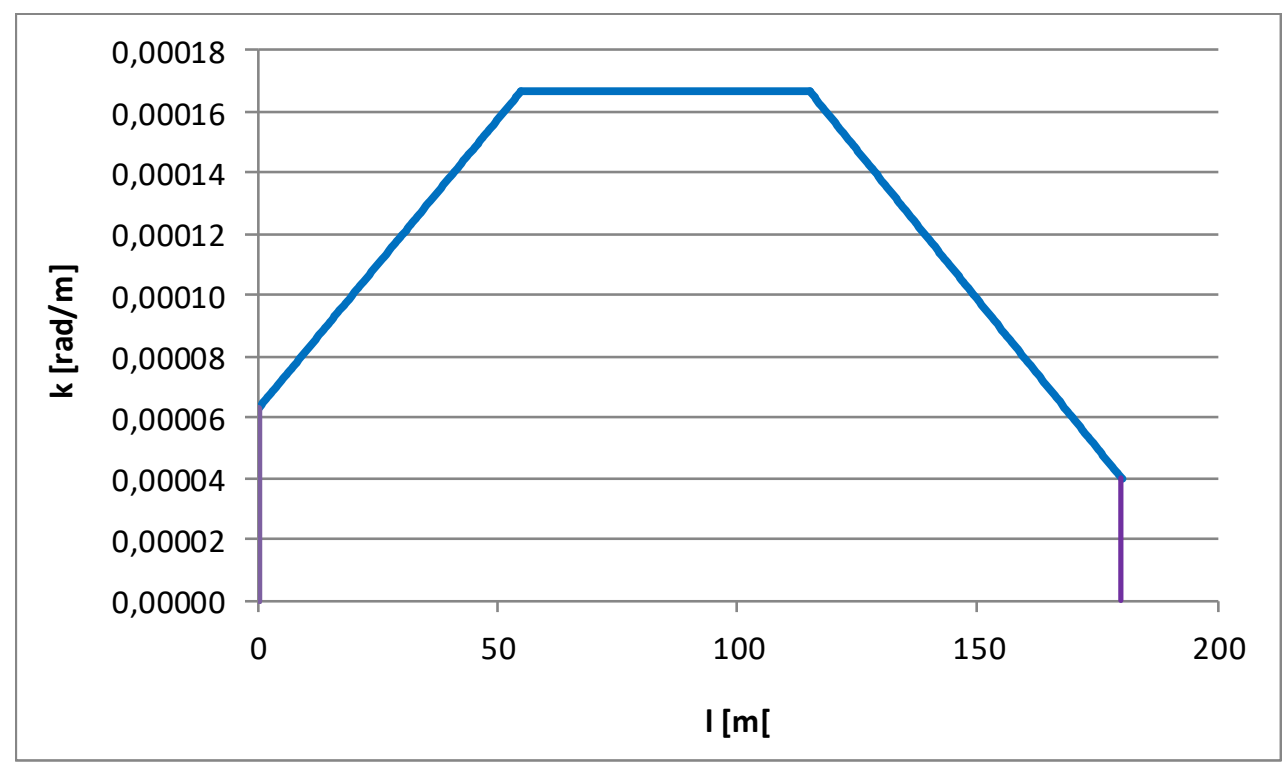

1. Curvature diagram with segments of linear curvature along the turnout path of the crossover $\left(R_{1}=16000 \mathrm{~m}, l_{1}=55 \mathrm{~m}, R_{2}=6000 \mathrm{~m}, l_{2}=60 \mathrm{~m}, l_{3}=65 \mathrm{~m}, R_{3}=25000 \mathrm{~m}\right)$

Modelling the curvature along the turnout path of the crossover makes possible to create an analytical record in the form of a function $k(l)$, where parameter $l$ determines position of a given point on the length of the curve. We can save the coordinate equations of the searched connection in a parametric form [3]:

$$
\begin{aligned}
& x(l)=\int \cos \Theta(l) d l \\
& y(l)=\int \sin \Theta(l) d l
\end{aligned}
$$

The tangent angle function $\Theta(l)$ is defined according to the equation:

$$
\Theta(l)=\int k(l) d l
$$

Commonly used, among others in commercial design support programs [1, 7], way of determining coordinates $x(l)$ i $y(l)$ is numerical integration of function $\cos \Theta(l)$ and $\sin \Theta(l)$. From a practical point of view, it gives sufficient accuracy. However, this method has one fundamental weakness and each geometrical case must be considered separately and the introduction of any generalization is very difficult here. If we want to get an opportunity to look at the problem more broadly, we should have an analytical method which is of general and full character.

An analytical solution to the problem of the application of linear curvature segments, i.e. the so-called "clotoid sections", is presented in [4]. It would be worthwhile to consider the application of sections of non-linear curvature and this issue was devoted to this study. Knowledge of the appropriate analytical formulas made it possible to conduct a comparative analysis of selected variants in the final part of the work, both with sections of linear and nonlinear curves.

\section{Analytical solution for the relief of curvature in extreme zones of crossover}

The course of curvature shown in Figure 1 is not, of course, the ideal solution. It would be worth considering the possibility of softening the entry into the turnout arc on its both sides 
and - in addition - resetting the curvature value at the extreme points of the geometrical system.

\section{Solving the problem for the initial zone}

We assume the boundary conditions

$$
\begin{cases}k(0)=k_{1} & k\left(l_{1}\right)=k_{2} \\ k^{\prime}(0)=C \frac{k_{2}-k_{1}}{l_{1}} & k^{\prime}\left(l_{1}\right)=0\end{cases}
$$

and differential equation

$$
k^{(4)}(l)=0
$$

assuming a numerical coefficient $C \geq 0$.

As a result of solving the differential problem (4), (5) we obtain the equation of curvature

$$
k(l)=k_{1}+\frac{C}{l_{1}}\left(k_{2}-k_{1}\right) l-\frac{2 C-3}{l_{1}^{2}}\left(k_{2}-k_{1}\right) l^{2}+\frac{C-2}{l_{1}^{3}}\left(k_{2}-k_{1}\right) l^{3}
$$

The tangent angle function $\Theta(l)$ is described by a dependence

$$
\Theta(l)=k_{1} l+\frac{C}{2 l_{1}}\left(k_{2}-k_{1}\right) l^{2}-\frac{2 C-3}{3 l_{1}^{2}}\left(k_{2}-k_{1}\right) l^{3}+\frac{C-2}{4 l_{1}^{3}}\left(k_{2}-k_{1}\right) l^{4}
$$

If we want to get a monotonic course of curvature $k(l)$ along the entire length of the curve, then the derivative $k^{\prime}(l)$ must be positive and at the same time the second derivative $k^{\prime \prime}(l)$ must be negative for $l \in\left\langle 0, l_{1}\right\rangle$.

$$
\begin{gathered}
k^{\prime}(l)=\frac{C}{l_{1}}\left(k_{2}-k_{1}\right)-\frac{4 C-6}{l_{1}^{2}}\left(k_{2}-k_{1}\right) l+\frac{3(C-2)}{l_{1}^{3}}\left(k_{2}-k_{1}\right) l^{2} \geq 0 \\
k^{\prime \prime}(l)=-\frac{4 C-6}{l_{1}^{2}}\left(k_{2}-k_{1}\right)+\frac{6(C-2)}{l_{1}^{3}}\left(k_{2}-k_{1}\right) l \leq 0
\end{gathered}
$$

As you can see, the course of the function $k^{\prime \prime}(l)$ is linear, so fulfilling the condition means that it is valid at both ends of the interval, i.e. $k^{\prime \prime}(0) \leq 0$ i $k^{\prime \prime}\left(l_{1}\right) \leq 0$. It follows that the parameter $C$ must take values from the range $C \in\langle 1.5 ; 3\rangle$.

Due to the ratio of the length of the parametric curve to the length of the curve with linear curvature, i.e. generalized clotoid, the most preferred solution seems to be a parametric transition curve for the value of $C=1.5$. We receive from dependencies (6) and (7)

$$
\begin{gathered}
k(l)=k_{1}+\frac{3}{2 l_{1}}\left(k_{2}-k_{1}\right) l-\frac{1}{2 l_{1}^{3}}\left(k_{2}-k_{1}\right) l^{3} \\
\Theta(l)=k_{1} l+\frac{3}{4 l_{1}}\left(k_{2}-k_{1}\right) l^{2}-\frac{1}{8 l_{1}^{3}}\left(k_{2}-k_{1}\right) l^{4}
\end{gathered}
$$

At the end of the zone, for $l=l_{1}$, angle value $\Theta\left(l_{1}\right)=\frac{3 k_{1}+5 k_{2}}{8} l_{1}$. 
To determine parametric equations $x(l)$ i $y(l)$ the procedure in [3] is used. We save the function $\Theta(l)$ in the form

$\Theta(l)=A_{11} l+A_{12} l^{2}+A_{13} l^{4}$

where

$$
A_{11}=k_{1}, \quad A_{12}=\frac{3\left(k_{2}-k_{1}\right)}{4 l_{1}} \quad A_{13}=-\frac{k_{2}-k_{1}}{8 l_{1}^{3}}
$$

and develop $\cos \Theta(l)$ and $\sin \Theta(l)$ into the Taylor series using the Maxima program [6]. As it turns out, the equation obtained in practice $x(l)$ can be simplified much, because most of its expressions are irrelevant. We get the following parametric equations:

$$
\begin{gathered}
x(l)=l \\
y(l)=\frac{1}{2} A_{11} l^{2}+\frac{1}{3} A_{12} l^{3}-\frac{1}{24} A_{11}^{3} l^{4}-\frac{1}{10}\left(A_{11}^{2} A_{12}-2 A_{13}\right) l^{5}
\end{gathered}
$$

\section{Solving the problem for the middle zone}

In the circular arc zone, i.e. for $l \in\left\langle l_{1}, l_{1}+l_{2}\right\rangle$, there is a constant curvature

$$
k(l)=k_{2}
$$

Function $\Theta(l)$ for the assumed $\mathrm{C}=1,5$ is described by the dependence

$$
\Theta(l)=-\frac{3 k}{8} l_{1}+k_{2} l
$$

At the end of a circular arc, for $l=l_{1}+l_{2}$, angle $\Theta\left(l_{1}+l_{2}\right)=\frac{3 k_{1}+5 k_{2}}{8} l_{1}+k_{2} l_{2}$.

The diagram showing the location of a circular arc is shown in Figure 2. We take the length of the circular arc 1_ (2) (measured along the arc itself). Its radius is $l_{2}$ measured on the arc itself. Its radius is $R_{2}$, and slope of the tangent at the starting point $s_{1}=\tan \Theta\left(l_{1}\right)$. We determine the coordinates of the centre of the arc point $S\left(x_{S}, y_{S}\right)$.

$$
\begin{aligned}
& x_{S}=x\left(l_{1}\right)-\frac{s_{1}}{\sqrt{1+s_{1}^{2}}} R_{2} \\
& y_{S}=y\left(l_{1}\right)+\frac{1}{\sqrt{1+s_{1}^{2}}} R_{2}
\end{aligned}
$$




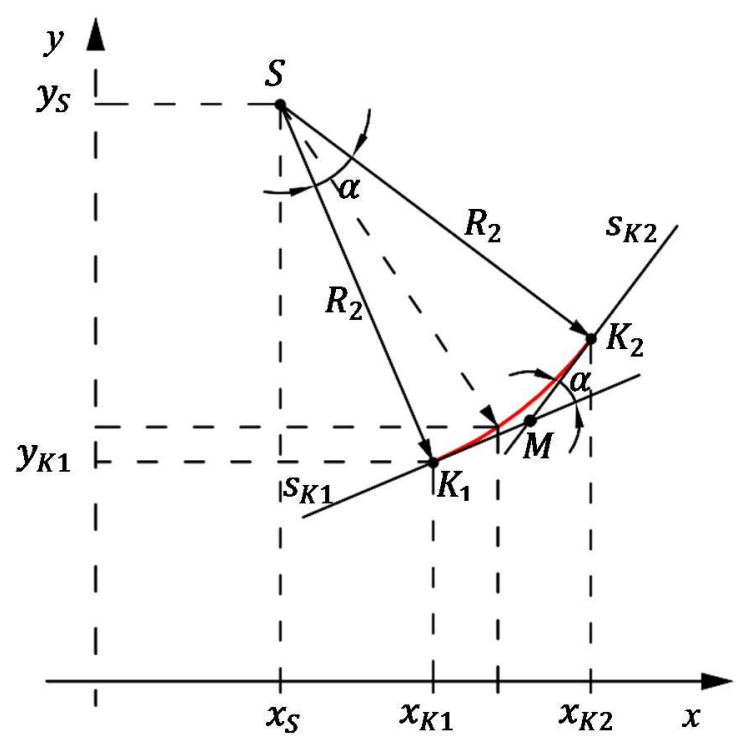

2. Diagram showing the location of a circular arc

The equation of a circular arc is as follows:

$$
y(x)=y_{S}-\left[R_{2}^{2}-\left(x_{S}-x\right)^{2}\right]^{\frac{1}{2}} \quad, x \in\left\langle x\left(l_{1}\right), x\left(l_{1}+l_{2}\right)\right\rangle
$$

The return angle of tangents is $\propto=\frac{l_{2}}{R_{2}}$. The angle of tangent of the arc at its end, i.e. for $x\left(l_{1}+l_{2}\right)$, is $\Theta\left(l_{1}+l_{2}\right)=\Theta\left(l_{1}\right)+\propto$, where is the value of the tangent slope at this point.

$$
s_{2}=\tan \left[\Theta\left(l_{1}\right)+\propto\right]
$$

To determine the coordinates of the end of a circular arc, you must first determine the coordinates of the point $M$ (Fig. 2); it is finally obtained

$$
\begin{aligned}
& x\left(l_{1}+l_{2}\right)=x\left(l_{1}\right)+\tan \frac{\alpha}{2}\left(\frac{1}{\sqrt{1+s_{1}^{2}}}+\frac{1}{\sqrt{1+s_{2}^{2}}}\right) R_{2} \\
& y\left(l_{1}+l_{2}\right)=y\left(l_{1}\right)+\tan \frac{\alpha}{2}\left(\frac{s_{1}}{\sqrt{1+s_{1}^{2}}}+\frac{s_{2}}{\sqrt{1+s_{2}^{2}}}\right) R_{2}
\end{aligned}
$$

\section{Solving the problem for the end zone}

We accept boundary conditions

$$
\begin{cases}k\left(l_{1}+l_{2}\right)=k_{2} & k\left(l_{1}+l_{2}+l_{3}\right)=k_{3} \\ k^{\prime}\left(l_{1}+l_{2}\right)=0 & k^{\prime}\left(l_{1}+l_{2}+l_{3}\right)=C \frac{k_{3}-k_{2}}{l_{3}}\end{cases}
$$

and differential equation (5). We assume that the numeric factor $C \geq 0$. The solution to the differential problem (5), (18) is as follows: 


$$
k(l)=c_{1}+c_{2} l+c_{3} l^{2}+c_{4} l^{3}
$$

where

$$
\begin{aligned}
& c_{1}=k_{2}+\left[\frac{3-C}{l_{3}^{2}}\left(l_{1}+l_{2}\right)^{2}+\frac{2-C}{l_{3}^{3}}\left(l_{1}+l_{2}\right)^{3}\right]\left(k_{3}-k_{2}\right) \\
& c_{2}=-\left[\frac{2(3-C)}{l_{3}^{2}}\left(l_{1}+l_{2}\right)+\frac{3(2-C)}{l_{3}^{3}}\left(l_{1}+l_{2}\right)^{2}\right]\left(k_{3}-k_{2}\right) \\
& c_{3}=\left[\frac{3-C}{l_{3}^{2}}+\frac{3(2-C)}{l_{3}^{3}}\left(l_{1}+l_{2}\right)\right]\left(k_{3}-k_{2}\right) \\
& c_{4}=-\frac{2-C}{l_{3}^{3}}\left(k_{3}-k_{2}\right)
\end{aligned}
$$

For $\mathrm{C}=1.5$ the values of these coefficients are

$$
\begin{aligned}
& c_{1}=k_{2}+\left[\frac{3}{2 l_{3}^{2}}\left(l_{1}+l_{2}\right)^{2}+\frac{1}{2 l_{3}^{3}}\left(l_{1}+l_{2}\right)^{3}\right]\left(k_{3}-k_{2}\right) \\
& c_{2}=-\left[\frac{3}{l_{3}^{2}}\left(l_{1}+l_{2}\right)+\frac{3}{2 l_{3}^{3}}\left(l_{1}+l_{2}\right)^{2}\right]\left(k_{3}-k_{2}\right) \\
& c_{3}=\left[\frac{3}{2 l_{3}^{2}}+\frac{3}{2 l_{3}^{3}}\left(l_{1}+l_{2}\right)\right]\left(k_{3}-k_{2}\right) \\
& c_{4}=-\frac{1}{2 l_{3}^{3}}\left(k_{3}-k_{2}\right)
\end{aligned}
$$

and the equation of the tangent inclination angle has the form

$$
\Theta(l)=c_{0}+c_{1} l+\frac{1}{2} c_{2} l^{2}+\frac{1}{3} c_{3} l^{3}+\frac{1}{4} c_{4} l^{4}
$$

where $c_{0}=\frac{3\left(k_{1}-k_{2}\right)}{8} l_{1}-\left[\frac{1}{2 l_{3}^{2}}\left(l_{1}+l_{2}\right)^{3}+\frac{1}{8 l_{3}^{3}}\left(l_{1}+l_{2}\right)^{4}\right]\left(k_{3}-k_{2}\right)$

Figures 3 and 4 show curvature graphs and tangent slope angles for values $C=1.5$ on the length of turnout of the crossroads having nonlinear curvature sections, and numerical characteristics corresponding to the turnout from Figure 1.

Parametric equations $x(l)$ i $y(l)$ for this zone, after the assumption $C=1.5$, is determined using the procedure outlined in [3]. We save the function $\Theta(l)$ in the form

$$
\Theta(l)=A_{31}+A_{32} l+A_{33} l^{2}+A_{34} l^{3}+A_{35} l^{4}
$$

where

$$
\begin{aligned}
& A_{31}=\frac{3\left(k_{1}-k_{2}\right)}{8} l_{1}-\left[\frac{1}{2 l_{3}^{2}}\left(l_{1}+l_{2}\right)^{3}+\frac{1}{8 l_{3}^{3}}\left(l_{1}+l_{2}\right)^{4}\right]\left(k_{3}-k_{2}\right) \\
& A_{32}=c_{1}=k_{2}+\left[\frac{3}{2 l_{3}^{2}}\left(l_{1}+l_{2}\right)^{2}+\frac{1}{2 l_{3}^{3}}\left(l_{1}+l_{2}\right)^{3}\right]\left(k_{3}-k_{2}\right)
\end{aligned}
$$




$$
\begin{aligned}
& A_{33}=\frac{1}{2} c_{2}=-\left[\frac{3}{2 l_{3}^{2}}\left(l_{1}+l_{2}\right)+\frac{3}{4 l_{3}^{3}}\left(l_{1}+l_{2}\right)^{2}\right]\left(k_{3}-k_{2}\right) \\
& A_{34}=\frac{1}{3} c_{3}=\left[\frac{1}{2 l_{3}^{2}}+\frac{1}{2 l_{3}^{3}}\left(l_{1}+l_{2}\right)\right]\left(k_{3}-k_{2}\right) \\
& A_{35}=\frac{1}{4} c_{4}=-\frac{1}{8 l_{3}^{3}}\left(k_{3}-k_{2}\right)
\end{aligned}
$$

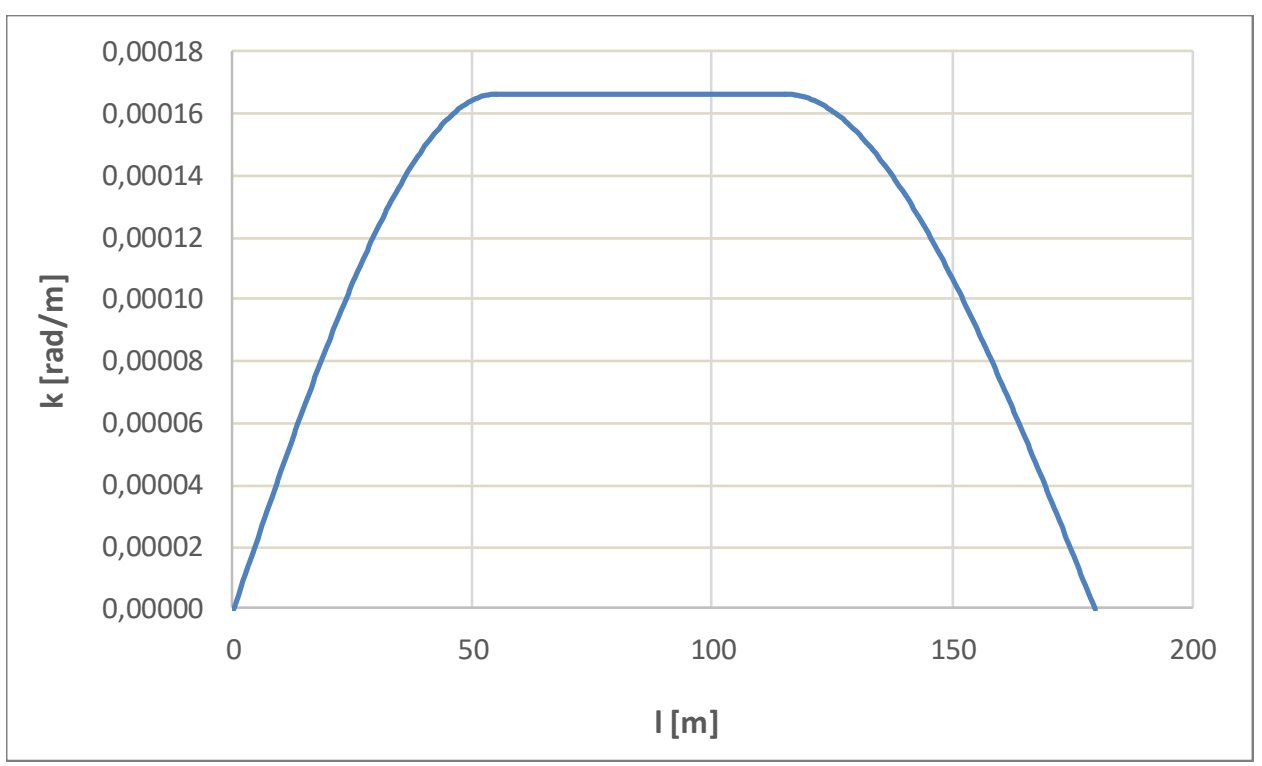

3. Curvature diagram with non-linear curvature segments for $C=1.5$ on the length of turnout of the turnout $\left(R_{1}=16000 \mathrm{~m}, l_{1}=55 \mathrm{~m}, R_{2}=6000 \mathrm{~m}, l_{2}=60 \mathrm{~m}, l_{3}=65 \mathrm{~m}, R_{3}=25000 \mathrm{~m}\right)$

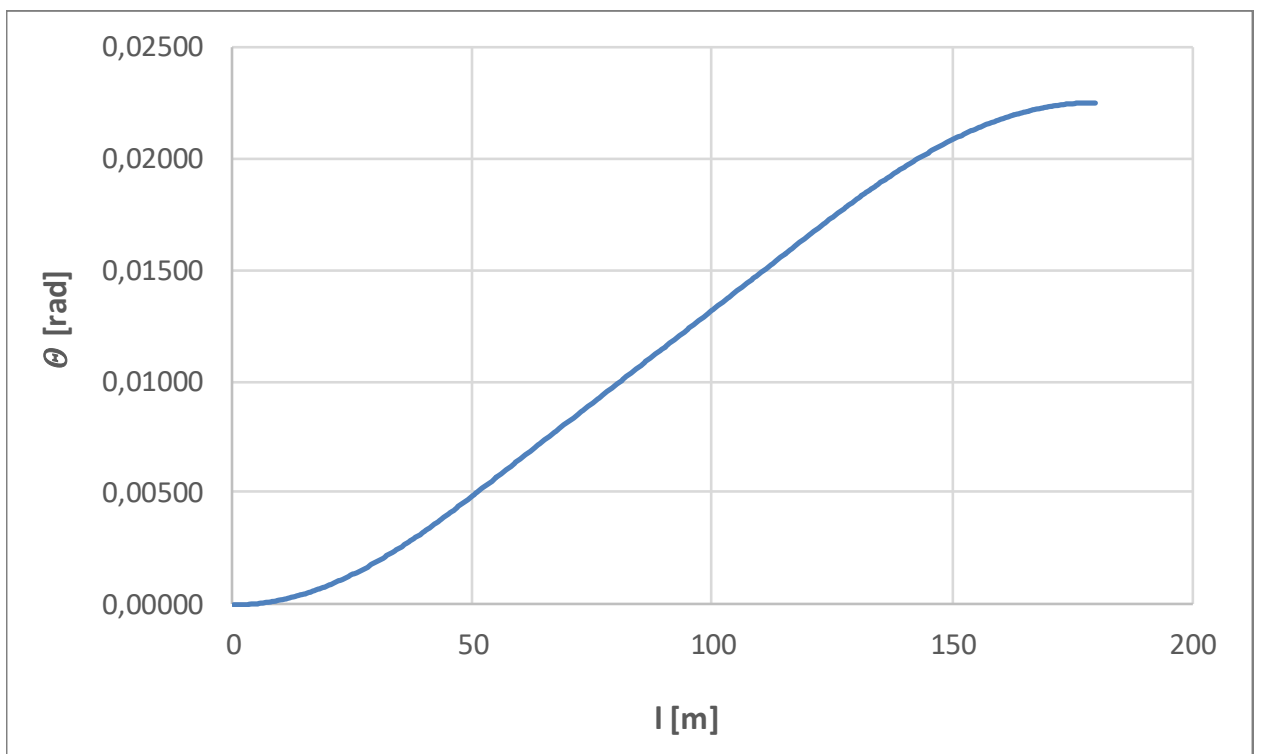

4. Graph of the tangent angle for $C=1.5$ on the length of turnout of the turnout from Figure $7\left(R_{1}=16000 \mathrm{~m}, l_{1}=55 \mathrm{~m}, R_{2}=6000 \mathrm{~m}, l_{2}=60 \mathrm{~m}, l_{3}=65 \mathrm{~m}, R_{3}=25000 \mathrm{~m}\right)$

After expanding the function $\cos \Theta(l)$ and $\sin \Theta(l)$ in the Taylor series using the Maxim program [6] and integration we get the following parametric equations: 


$$
x(l)=x\left(l_{0}\right)+\cos \Theta_{0}\left(l-l_{0}\right)-\frac{1}{2}\left(A_{32}+4 A_{35} l_{0}^{3}+3 A_{34} l_{0}^{2}+2 A_{33} l_{0}\right) \sin \Theta_{0}\left(l-l_{0}\right)^{2}
$$

$$
\begin{aligned}
& y(l)=y\left(l_{0}\right)+\sin \Theta_{0}\left(l-l_{0}\right)+\frac{1}{2}\left(A_{32}+4 A_{35} l_{0}^{3}+3 A_{34} l_{0}^{2}+2 A_{33} l_{0}\right) \cos \Theta_{0}\left(l-l_{0}\right)^{2} \\
& -\frac{1}{6}\left(A_{32}^{2}+8 A_{32} A_{35} l_{0}^{3}+6 A_{32} A_{34} l_{0}^{2}+4 A_{32} A_{33} l_{0}+16 A_{35}^{2} l_{0}^{6}+24 A_{34} A_{35} l_{0}^{5}+\right. \\
& \left.16 A_{33} A_{35} l_{0}^{4}+9 A_{34}^{2} l_{0}^{4}+12 A_{33} A_{34} l_{0}^{3}+4 A_{33}^{2} l_{0}^{2}\right) \sin \Theta_{0}\left(l-l_{0}\right)^{3} \\
& -\frac{1}{24}\left(A_{32}^{3}+12 A_{32}^{2} A_{35} l_{0}^{3}+9 A_{32}^{2} A_{34} l_{0}^{2}+6 A_{32}^{2} A_{33} l_{0}+48 A_{32} A_{35}^{2} l_{0}^{6}+72 A_{32} A_{34} A_{35} l_{0}^{5}+\right. \\
& 48 A_{32} A_{33} A_{35} l_{0}^{4}+27 A_{32} A_{34}^{2} l_{0}^{4}+36 A_{32} A_{33} A_{34} l_{0}^{3}+12 A_{32} A_{33}^{2} l_{0}^{2}+64 A_{35}^{3} l_{0}^{9}+144 A_{34} A_{35}^{2} l_{0}^{8}+ \\
& 96 A_{33} A_{35}^{2} l_{0}^{7}+108 A_{34}^{2} A_{35} l_{0}^{7}+144 A_{33} A_{34} A_{25} l_{0}^{6}+27 A_{34}^{3} l_{0}^{6}+48 A_{33}^{2} A_{35} l_{0}^{5}+54 A_{33} A_{34}^{2} l_{0}^{5}+ \\
& \left.36 A_{33}^{2} A_{34} l_{0}^{4}+8 A_{33}^{3} l_{0}^{3}-24 A_{35} l_{0}-6 A_{34}\right) \cos \Theta_{0}\left(l-l_{0}\right)^{4}
\end{aligned}
$$

where

$$
l_{0}=l_{1}+l_{2} \quad \Theta_{0}=\Theta\left(l_{1}+l_{2}\right)
$$

\section{Analysis of selected variants}

Variants considered in the work arose as a result of the introduction of three zones of curvature distribution along the turnout path of the crossover. In the central zone the curvature is constant, i.e. a circular arc is used here. In contrast, in the extreme zones curvature is variable, as is the case in the transition curve. The course of the curvature can be linear and the problem solution is then unambiguous [4] or non-linear, where different solutions are possible. This paper proposes a polynomial solution for the latter case. Of course, for both the linear and non-linear curves segments, various detailed cases are possible, related to the curvature values and the lengths of individual zones.

The theoretical relations given in this work and in [4] are of universal nature, i.e. they allow any curvature values to be accepted at the beginning and at the end of the geometrical system. Zero values can be of particular interest here. The general cases appearing in Figures 1 and 2 and the corresponding cases with zero curvature on both sides of the system, which were created after the admission, were subjected to a comparative analysis.

$k_{1}=k_{3}=0$.

- Turnout I (with sections of linear curvature)

$R_{1}=16000 \mathrm{~m}, l_{1}=55 \mathrm{~m}, R_{2}=6000 \mathrm{~m}, l_{2}=60 \mathrm{~m}, l_{3}=65 \mathrm{~m}, R_{3}=25000 \mathrm{~m}$

- Turnout II (with sections of linear curvature) $R_{1}=\infty, l_{1}=55 \mathrm{~m}, R_{2}=6000 \mathrm{~m}, l_{2}=60 \mathrm{~m}, l_{3}=65 \mathrm{~m}, R_{3}=\infty$

- Turnout III (with sections of nonlinear curvature) $R_{1}=16000 \mathrm{~m}, l_{1}=55 \mathrm{~m}, R_{2}=6000 \mathrm{~m}, l_{2}=60 \mathrm{~m}, l_{3}=65 \mathrm{~m}, R_{3}=25000 \mathrm{~m}$

- Turnout IV (with sections of nonlinear curvature) $R_{1}=\infty, l_{1}=55 \mathrm{~m}, R_{2}=6000 \mathrm{~m}, l_{2}=60 \mathrm{~m}, l_{3}=65 \mathrm{~m}, R_{3}=\infty$

The results of calculations of characteristic values of the return path of the mentioned turnouts are presented in the Table 1. Compound curvature plots on the length are shown in Figure 5. 
Tab. 1. List of characteristic values for compared turnouts

\begin{tabular}{|c|c|c|c|c|c|c|c|c|c|}
\hline & \multicolumn{3}{|c|}{$l=l_{1}$} & \multicolumn{3}{c|}{$l=l_{1}+l_{2}$} & \multicolumn{3}{c|}{$l=l_{1}+l_{2}+l_{3}$} \\
\cline { 2 - 9 } & $\begin{array}{c}x(l) \\
{[\mathrm{m}]}\end{array}$ & $\begin{array}{c}y(l) \\
{[\mathrm{m}]}\end{array}$ & $\begin{array}{c}Q(l) \\
{[\mathrm{rad}]}\end{array}$ & $\begin{array}{c}x(l) \\
{[\mathrm{m}]}\end{array}$ & $\begin{array}{c}y(l) \\
{[\mathrm{m}]}\end{array}$ & $\begin{array}{c}Q(l) \\
{[\mathrm{rad}]}\end{array}$ & $\begin{array}{c}x(l) \\
{[\mathrm{m}]}\end{array}$ & $\begin{array}{c}y(l) \\
{[\mathrm{m}]}\end{array}$ & $\begin{array}{c}Q(l) \\
{[\mathrm{rad}]}\end{array}$ \\
\hline I & $\begin{array}{c}54,999 \\
3\end{array}$ & 0,147 & 0,00630 & 114,995 & 0,825 & 0,01630 & 179,982 & 2,148 & 0,02302 \\
\hline II & $\begin{array}{c}54,999 \\
9\end{array}$ & 0,084 & 0,00458 & 114,997 & 0,659 & 0,01458 & 179,986 & 1,842 & 0,02000 \\
\hline III & $\begin{array}{c}54,999 \\
6\end{array}$ & 0,165 & 0,00702 & 114,995 & 0,887 & 0,01702 & 179,979 & 2,291 & 0,02476 \\
\hline IV & $\begin{array}{c}54,999 \\
7\end{array}$ & 0,113 & 0,00573 & 114,996 & 0,757 & 0,01573 & 179,983 & 2,061 & 0,02250 \\
\hline
\end{tabular}

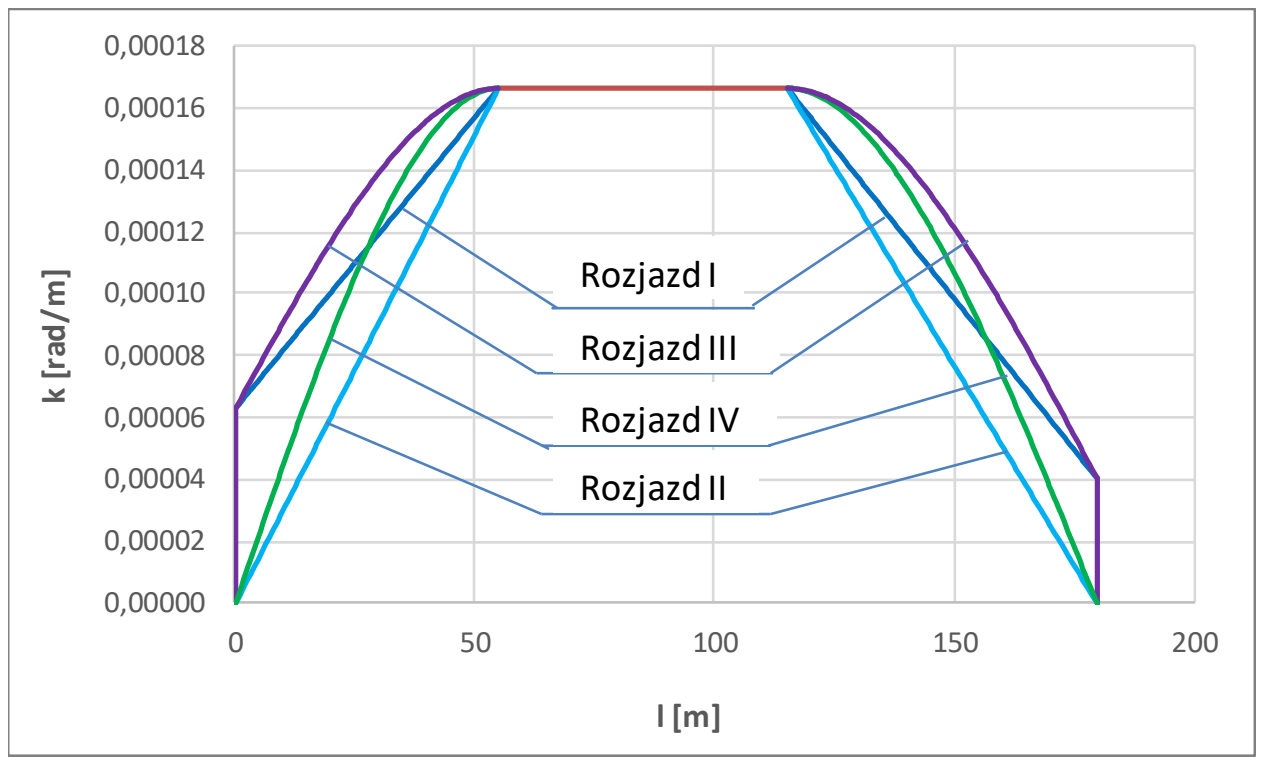

5. Curve diagrams on the length of the return path of the compared turnouts $\left(R_{1}=16000 \mathrm{~m} / \infty, l_{1}=55 \mathrm{~m}, R_{2}=6000 \mathrm{~m}, l_{2}=60 \mathrm{~m}, l_{3}=65 \mathrm{~m}, R_{3}=25000 \mathrm{~m} / \infty\right)$

Currently, the most common type of crossover with segments of variable linear curvature is turnout I. The linear curvature along the entire length of the extreme zones in the 2 nd junction is the most deviating from the other cases. Turnout III is the closest to turnout I, and its main advantage is the lack of bends in the curvature diagram at the beginning and end of the circular arc. Particularly noteworthy is the turnout IV. In addition to the gentle transition in the extreme regions of the circular arc, it is characterized by zero curvature at both ends of the geometrical system. Perhaps this solution should replace the use of "clotoid sections". The dynamic analysis presented in paper [5] indicates the benefits resulting from its use. However, this requires further analysis, but there is no doubt that the method presented in this work allows for creating many different solutions. Figure 6 shows charts of horizontal ordinates over the length of the analysed turnouts. 


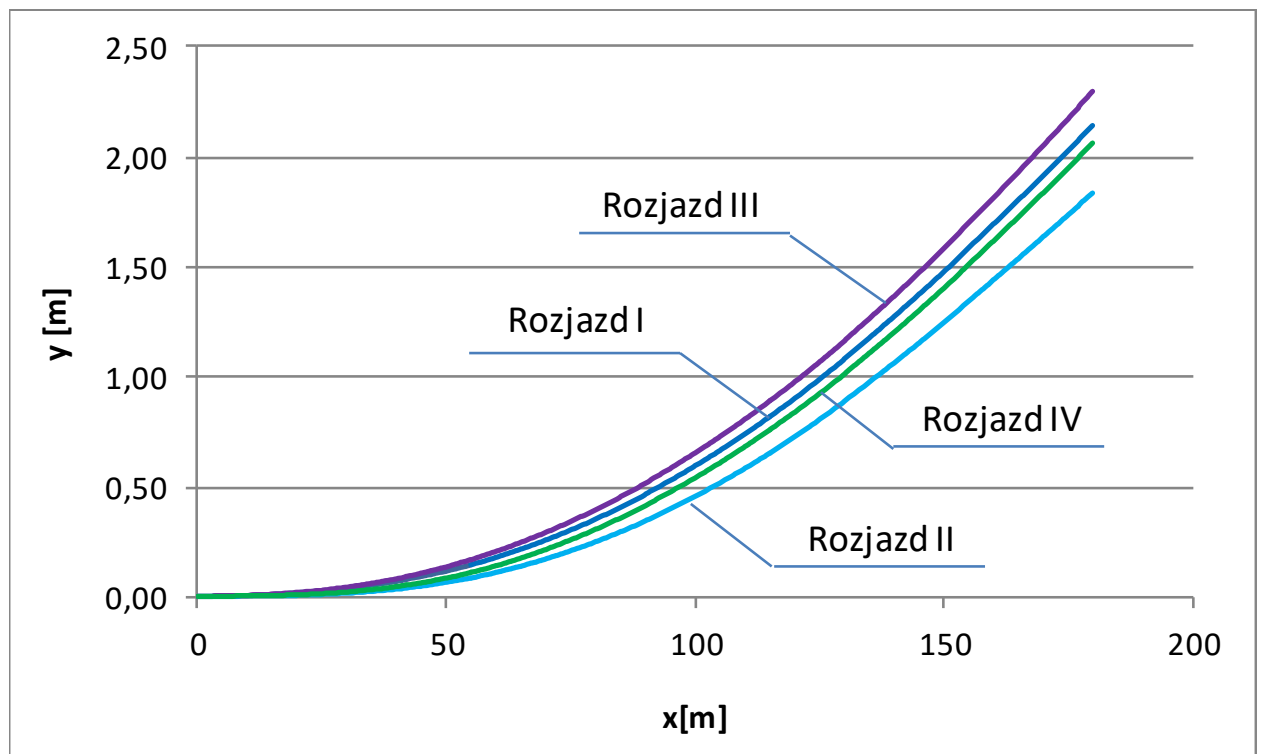

6. Plots of horizontal ordinates on the length of the return path of the compared turnouts $\left(R_{1}=\right.$ $\left.16000 \mathrm{~m} / \infty, l_{1}=55 \mathrm{~m}, R_{2}=6000 \mathrm{~m}, l_{2}=60 \mathrm{~m}, l_{3}=65 \mathrm{~m}, R_{3}=25000 \mathrm{~m} / \infty\right)$

\section{Summary}

In the reverse track of a typical railway junction, called ordinary, a single circular arc is used without transients. As a result, there are places of rapid, abrupt change in the ordinates of the curvature diagram at the beginning and end of the turnout. In recent years, in some countries, aiming at smoothing the curvature graph in these regions, the so-called "Clothoid sections" on both sides of a circular arc on which the curvature changes in a linear manner.

In the case of switches with variable curvature due to the individual nature of their design it is possible to adjust the given turnout to local requirements. This is particularly important in parallel tracks connections, where the main issue is maintaining the proper track gauge. The decisive role here is played by the final elevation of the reverse path, which can be shaped in switches with variable curvature by manipulating the slant and the crossover radius.

Numerical integration of functions $\cos \Theta(l)$ i $\sin \Theta(l)$ is commonly used for determining coordinates $x(l)$ i $y(l)$ in switches with variable curvature. Each geometric case must therefore be considered separately and the introduction of any generalization is very difficult here. This work presents an analytical method of solving the problem, which is general and full. Appropriate equations for the case of proposed sections of non-linear curvature have been determined. In the comparative analysis of selected variants, the dependencies for segments of linear curvature presented in [4] were also used.

The theoretical relations are of universal nature, i.e. they allow any curvature values to be accepted at the beginning and at the end of the geometrical system. Zero values can be of particular interest here. A comparative analysis of selected variants indicated a favourable course of curvature in the non-linear variant, which in addition to the smooth transition in the extreme regions of the circular arc is characterized by zero curvature at both ends of the geometric system. Perhaps this solution should replace the use of the so-called "Clotoid sections".

\section{Source materials}

[1] AutoCAD, http://www.autodesk.pl

[2] Fei Weizhu: Major technical characteristics of high-speed turnout in France. Journal of Railway Engineering Society, vol. 26, no. 9, pp. 18-35, 2009.

[3] Koc W.: Analytical method of modelling the geometric system of communication route. Mathematical Problems in Engineering, vol. 2014, Article ID 679817, 2014. 
[4] Koc W.: Kształtowanie toru zwrotnego rozjazdu z odcinkami krzywizny liniowej (artykuł złożony w redakcji czasopisma naukowego)

[5] Koc W., Palikowska K.: Dynamic analysis of the turnout diverging track for HSR with variable curvature sections. World Journal of Engineering and Technology, vol. 5, pp. 42-57, 2017.

[6] Maxima package, http://maksima.sourceforge.net

[7] MicroStation, http://www.bentley.com

[8] Plank B.: Linie dużych prędkości realizowane przez VAE. Prezentacja firmy Voestalpine GmbH, 2007.

[9] Technical Memorandum: Alignment design standards for high-speed train operation. Prepared by Parsons Brinckerhoff for the California High-Speed Rail Authority, USA, 2009.

[10] Wang P.: Design of high-speed railway turnouts. Theory and Applications. Elsevier Science \& Technology, Oxford, United Kingdom, 2015. 